\title{
AUTOMEDICAÇÃO POR PROFISSIONAIS E ACADÊMICOS DA ÁREA DA SAÚDE: uma revisão de literatura
}

\author{
Deyverson Ricardo Pereira de SOUZA ${ }^{1}$
}

Maria ESMÉRIA NETA ${ }^{2}$

\author{
${ }^{1}$ Residência em Saúde da Família, ricardodeyverson@gmail.com. \\ ${ }^{2}$ Especialista em Urgência e Emergência, Professora da Associação Educativa do Brasil, esmerianeta@ yahoo.com.br.
}

Recebido em: 10/04/2016 - Aprovado em: 24/10/2016 - Disponibilizado em: 18/12/2016

\begin{abstract}
RESUMO:
A automedicação está presente no contexto dos profissionais e acadêmicos da saúde e representa um sério problema de saúde dos muitos que a profissão da saúde possui. Com isso, este trabalho se propôs a descrever o problema identificando suas causas, a que estão relacionados e os medicamentos mais utilizados. A metodologia utiliza foi à revisão de literatura através do banco de dados da Scientific Eletronic Library On-Line (SciELO) e Literatura LatinoAmericana e do Caribe em Ciências da Saúde (LILACS) sendo selecionados no idioma português, produção dos anos de2003 a 2013 e relação do tema de acordo com o resumo dos artigos. Foram encontrados 48 artigos, porém através dos critérios pré-estabelecidos foram selecionados 10 artigos pela relação com o tema. Os resultados apontam as dores e estresse como fatores precipitantes e analgésicos e antinflamatórios como medicamentos mais utilizados.

Palavras-Chave: Medicação. Autocuidado. Automedicação. Profissionais da saúde. Acadêmicos de saúde.
\end{abstract}

\begin{abstract}
:
Self-medicationis present in the context of professional and academic health and represents a serious health problem that many of the health profession have. Therefore, this study aimed to describe the problem by identifying its causes, to which they are related and the most widely used drugs. The methodology used was the literature revision through the database of the Scientific Electronic Library Online (SciELO) and Latin American and Caribbean Health Sciences (LILACS) were selected by the national language, year of production 2003 until 2013 and the theme according to the summary of the articles. 48 articles were found, however through the pre-established rules 10 articles were selected by the relationship with the subject. The results show the pain and stress as precipitating factors and analgesics and antiinflammatory drugs as the most used.
\end{abstract}

Keywords: Medication. Self-care.Self-medication .Health professionals. Health academic.

\section{INTRODUÇÃO}

A automedicação constituisena seleção e o uso de medicamentos por pessoas para tratar doenças ou sintomas autodiagnosticados e deve ser entendida como um dos elementos do autocuidado. Esta prática é bastante difundida não apenas no Brasil, mas também em outros países, em decorrência dos sistemas de saúde precários, que acabam por induzir os pacientes a recorrerem a meios não corretos para o alívio de sintomas que os afligem.Por um lado, os medicamentos podemaumentar a expectativa de vida, erradicar certas doenças,trazer benefícios sociais e econômicos, no entanto por outro lado podem aumentar os custos da atenção à saúde se utilizados inadequadamente e ou levar à ocorrência de reações adversas. As reações se tornam o grande problemada automedicação (SANTOS et al., 2012).

As formas de automedicação são múltiplas: adquirir o medicamento sem receita, compartilhar 
remédios com membros da família ou do círculo social, desviar unidades de receitas destinadas a outra terapêutica, reutilizar antigas prescrições e descumprir orientação profissional, prolongando ou interrompendo precocemente a posologia e o período de tempo indicados na receita. Nesses casos, a eficiência terapêutica pode ser comprometida devido a uma provável compreensão das bases patológicas da doença e do real mecanismo de ação da droga por parte de leigos que utilizam a automedicação (FONSECA et al., 2010).

É fato que os medicamentos são um componente essencial da terapêutica e que desempenhamum reconhecido papel na recuperação e na manutenção da saúde e no bem-estar geral dos usuários desses produtos. Por outro lado, a crençaexcessiva em seu poder terapêuticoos levouà condição de mercadoria especial, lastreada peloconhecimento científico como único modo válido de se obter saúde (LUZ et al., 2012).

Com a propaganda massiva e a facilidade de acesso a medicamentos em farmácias e supermercados subentendeuma impressão de que são livres de riscos. Além disso, estimula o uso indiscriminado, o que nem sempre resulta nos efeitos esperados pelos indivíduos, e expõem estes consumidores a reações indesejadas, às reações adversas, sempre crescentes devido ao consumo elevado de medicamentos que se observa atualmente. A crença de que a pílula seja capaz de eliminar ou, pelo menos, amenizar estes problemas é fator complementar e primordial para o consumo (AQUINO; BARROS; SILVA, 2010).

A origem da automedicação dá-se com o surgimento de dores, principalmente associadas a dores de cabeça ou algum desconforto que precipita um desarranjo no bem-estar biopsicossocial (SOUZA, 2011).

Como em todo trabalho, a profissão dos profissionais da saúde pode comprometer o processo de viversaudável dos seus trabalhadores devido estresse, carga horária de trabalho, acumulo de funções e entre outros. Por isso, para obter oalívio ou almejando a cura de transtornos físicos ou psíquicos, inúmeros profissionais de enfermagem recorrem, com frequência, ao uso de terapias medicamentosas,principalmente utilizando-se da automedicaçãoo que representa uma conduta diametralmente oposta ao conhecimento que esses profissionais de saúde possuem quanto ao uso indiscriminado de medicamentos (BAGGIO; FORMAGGIO, 2009).

Seguindo a linha de pensamento do uso incorreto de medicamentos, o profissional, ao submeter-se ao uso inicial depsicotrópicos, por exemplo, 
frequentemente, prosseguecom outras experiências descontroladas, revelando mudanças comportamentais. Senso assim, seu perfilmodificado passa a desvelar um desempenho indesejável: baixo rendimento, altos índices de absenteísmo, não colaborativo com a equipe, convivência difícil edesconforto social no ambiente de trabalho, além de ser susceptível a erros e acidentes (DIAS et al., 2011).

Com a automedicação é possível obter, mesmo que temporariamente, o alívio aos sintomas físicos ou psíquicos apresentados. No entanto, o apelo do profissional de enfermagem para a autoterapia farmacológica - uso sem prescrição médica de analgésicos, anti-inflamatórios e, principalmente, psico-fármacos, remete aos riscos de intoxicações, reações alérgicas einterações medicamentosas (BAGGIO; FORMAGGIO, 2009).

Portanto, a prática de autocuidado fica evidente através do uso de fármacos para paliar ou buscar cura para algum desconforto vivenciado por um profissional ou acadêmico da área da saúde,e este estudo procura fornecer informações sobre a prática da automedicação e características da mesma através de revisão da literatura. Assim, facilita o processo de ações de intervenção no contexto dos profissionais de saúde o que, também, alerta aos próprios sujeitos a conscientizarem a respeito do assunto. Com isso, este trabalho se propôs a descrever o problema identificando suas causas, a que estão relacionados e os medicamentos mais utilizados.

\section{METODOLOGIA}

Trata-se de uma revisão literária sobre o tema “Automedicação por acadêmicos e profissionais da área da saúde: uma revisão literária" onde foi utilizada a base de dados da Scientific Eletronic Library Online (SciELO) e Literatura Latino-America e do Caribe em Ciências da Saúde (LILACS), Brasil, pesquisa de artigos na Biblioteca Virtual em Saúde (BVS). Foram utilizados os seguintes descritores: "autocuidado, automedicação e profissionais da saúde", para pesquisa da bibliografia de interesse, sendo excluídos os artigos estrangeiros, ou seja, somente artigos escritos no idioma portuguêsBrasil.

De acordo com a busca proposta pelos autores foram encontrados 48 artigos de acordo com os descritores, sendo estes procedentes de vários estudos diferentes com propostas diversas. Procedeu-se então à leitura dos resumos para identificar os artigos que possuíam relação com o tema em estudo, nessa etapa os pode-se delimitar qual a literatura a ser estudada em profundidade a partir dos objetivos, metodologia de estudo e resultados dos autores. 
Dentre os artigos encontrados foram selecionados 10 pertinentes ao tema de interesse de pesquisado estudo em questão. Além disso, a pesquisa restringiu-se ao espaço de tempo de 10 anos, ou seja, de artigos publicados entre os anos de 2006 a 2016 para que os dados pesquisados fossem o mais recente possível com uma amostra adequada. Por se tratar de revisão de literatura não foi necessária a submissão ao comitê de ética, haja vista não envolver pesquisa com seres humanos nem ser de dados particulares, pois os documentos pesquisados possuem caráter de ordem pública.

\section{RESULTADOS E DISCUSSÃO}

Foram incluídos artigos indexados nos bancos de dados Scientific Library Online (SciELO) e Literatura Latino Americana e do Caribe em Ciência da Saúde (LILACS), pois estes bancos de dados são específicos da área da saúde, possuem quantidades suficientes de publicações em vários temas e possuem, em sua grande maioria, literaturas de ordem pública e gratuita. A partir do enfoque Automedicação por Profissionais e Acadêmicos da Área da Saúde, procedeu-se à leitura dos resumos dos artigos com o objetivo de alcançar aqueles que convergiam ao tema proposto e davam subsídio para o desenvolvimento do estudo a ser realizado com dados que fossem suficientes para tal sendo, portanto observados os resultados dos artigos e sua metodologia.

Após a leitura estabeleceuse o assunto, sendo este:“Automedicação por Profissionais e Acadêmicos da Área da Saúde". Forambuscados nos artigos os seguintes assuntos: área do conhecimento; delineamento metodológico; variáveis analisadas; e principais resultados.

Em relação aos locais de realização dos estudos dos artigos são apresentados na tabela a seguir:

Tabela 1 - Distribuição dos artigos segundo o local de realização do estudo

\begin{tabular}{lll}
\hline Local & $\mathbf{N}^{\mathbf{0}}$ de Estudos & $\mathbf{\%}$ \\
\hline Belo Horizonte, & $\mathbf{1}$ & $\mathbf{1 0}$ \\
MG & & \\
São Paulo, SP & $\mathbf{1}$ & $\mathbf{1 0}$ \\
Pelotas, RJ & $\mathbf{1}$ & $\mathbf{1 0}$ \\
Santos, SP & $\mathbf{1}$ & $\mathbf{1 0}$ \\
Recife, PE & $\mathbf{2}$ & $\mathbf{2 0}$ \\
Rio de Janeiro, RJ & $\mathbf{2}$ & $\mathbf{2 0}$ \\
Alfenas, MG & $\mathbf{1}$ & $\mathbf{1 0}$ \\
Outra & $\mathbf{1}$ & $\mathbf{1 0}$ \\
\hline
\end{tabular}

Fonte: Artigos utilizados para redação deste estudo.

Com vistas ao espaço geográfico de realização dos 10 estudos, $70 \%$ deles encontram-se na região Sudeste do país sendo $30 \%$ no estado do Rio de Janeiro, e $20 \%$ nos estados de São Paulo e Minas Gerais sendo concentrados, principalmente em suas respectivas capitais, com exceção da cidade de Santos-SP e Alfenas-MG onde em cada uma foi desenvolvidos 1 estudo. Os demais 
estudos foram realizados no Nordeste $(20 \%$, em Recife-PE) e Região Central (10\%, Goiás). O que possa ser deduzido que há uma produção maior de estudos na região sudeste, tendo em vista o tema. Uma das limitações desta pesquisa éaausência determinação de razões pelas quais os estudos são em sua maioria realizados na região sudeste.

Os sinais e sintomas que aparecem com maior frequência são condensados neste próximo quadro apresentando o número em que estes sinais e sintomas aparecem na literatura em estudo.

Tabela 2 - Automedicaçãorelacionada a sinais e/ou sintomasconformea citação nos artigos

\begin{tabular}{lll}
\hline Sinal/Sintoma & $\mathbf{N}^{\mathbf{0}}$ & $\mathbf{\%}$ \\
\hline Dor & 4 & 40 \\
Febre & 2 & 20 \\
Resfriado & 2 & 20 \\
Problemas com a & 2 & 20 \\
garganta & & \\
Cansaço/Estresse & 3 & 30 \\
Redução da Obesidade & 1 & 10 \\
Processo alérgico & 1 & 10 \\
\hline
\end{tabular}

Fonte: Artigos utilizados para redação deste estudo.

No processo da automedicação o ponto precipitante parece estar quase sempre associado à dor (40\%) explicitada como causa ou justificativa desse processo. São também citados o cansaço e estresse em $30 \%$ da literatura analisada e febre, resfriado e problemas de garganta em $20 \%$ do material, eprocesso alérgico e redução da obesidade são a causa em $10 \%$ dos artigos. Com isso, pode ser observado que o uso do medicamento sem prescrição e/ou orientação corretas é seguido de queixas, principalmente relacionadas à dor. $\mathrm{O}$ cansaço e o estresse aparecem em segundo lugar em relação à pesquisa.

A partir das justificativas utilizadas pelos sujeitos como razão da automedicação os fármacos correspondentes são apresentados segundo sua evidência nos artigos. Os números da tabela subsequente referem-se à frequência em que são citados nos estudos.

Tabela 3 - Principaismedicamentoscitadosnos artigos utilizadospara automedicação

\begin{tabular}{lll}
\hline Medicamento & $\mathbf{N}^{\mathbf{0}}$ de Citações & $\%$ \\
\hline Analgésicos & 8 & 80 \\
Antinflamatório & 7 & 70 \\
Antipiréticos & 3 & 30 \\
Antibióticos & 3 & 30 \\
Psicotrópicos & 3 & 30 \\
Anti-Obesidade & 1 & 10 \\
Antialérgico & 1 & 10 \\
\hline
\end{tabular}

Fonte: Artigos utilizados para redação deste estudo.

Os principais fármacos utilizados são analgésicos sendo relacionados (80\%), quase sempre, à condição de dor, $70 \%$ dos artigos citam os antinflamatórios sendo utilizados na automedicação, e antipiréticos, antibióticos e psicotrópicos são descritos em $30 \%$ dos artigos e em 10\% dos estudos aparecem medicamentos utilizados para antiobesidade e antialérgicos. 
Tema 1: As principais causas da automedicação.

A prática da automedicação não está restrita apenas aos leigos, ao contrário, é muito difundida aos profissionais médicos e enfermeiros, pois estes estão em contato constante $\mathrm{e}$ ter fácil acesso às medicações. Apesar de estarem cientes dos riscos envolvidos ignoram assumindo essa prática sentindo-se seguros por estarem em um ambiente hospitalar e este comportamento fazer parte do convívio social. A informação obtida através dos estudos na área da saúde parece estar associada a uma convicção de que tal prática estaria respaldada de alguma maneira. Isto também pode ser observado entre os acadêmicos da área da saúde (SANTOS et al., 2012).

É conhecido que o uso de substâncias psicoativas pode se tornar indiscriminado quando o usuário, neste caso, o próprio profissional perde o controle sobre a droga. O uso de uma droga pode ocasionar dependência farmacológica e, mesmo vivendo em condições favoráveis, o sujeito poderá recorrer às drogas para vencer a monotonia do cotidiano, combater a ansiedade, a depressão, a dor ou simplesmente para obter prazer (BAGGIO;FOMAGGIO, 2009).

\section{Procurar a farmácia é a} primeira escolha na resolução de problemas de saúde, bem como a maioria dos medicamentos consumidos que são vendidos sem receitas, principalmente em países com problemas estruturais no sistema de saúde. E também, muitos dos países industrializados apresentam farmácias, drogarias e supermercados que disponibilizam os medicamentos mais comuns sem apresentação do receituário (BRITO, 2010).

Numa análise feita em um grupo de acadêmicos sobre a utilização de medicamentos para alívio da dor, a maior parte dos acadêmicos entrevistados que se automedicavam $(54,1 \%)$ referiram o uso a partir de julgamento próprio e outros (34,9\%) atendiam a opinião de familiares, ou seja, além do fator sintomático a influência de outras pessoas pode contribuir para a automedicação. Além disso, alguns dos entrevistados justificaram não possuir tempo para ir ao médico e que o acesso ao balconista é bem mais barato, prático e rápido. $\mathrm{O}$ que chama a atenção desse fato é ver que a "prescrição" dos medicamentos está sendo feita por pessoas inabilitadas para tal (SOUZA, 2011).

O uso de medicamento para redução de peso é descrito em uma pesquisa com acadêmicos do curso de medicina onde $20 \%$ dos que utilizavam algum método medicamento para emagrecer $49 \%$ o faziam por automedicação e o seu uso está diretamente ligado a um período mais avançado do curso, ou seja, os acadêmicos estudados mostraram que aqueles que eram do $1^{\circ}$ ano do curso de graduação de medicina 
que faziam uso de medicação para a redução do peso $14 \%$ deles faziam uso sem prescrição médica, já em relação aos do $5^{\circ}$ e $6^{\circ}$ anos demonstram que os praticantes de automedicação para redução do peso eram em uma proporção dobrada comparado aos alunos iniciantes do curso pois eram de $28 \%$ e $27 \%$ respectivamente. Isso demonstra que os acadêmicos utilizam medicamentos sem prescrição em maior proporção associando a um conhecimento que é adquirido no decorrer do curso (FIQUEIREDO, 2009).

$\mathrm{Da}$ mesma forma, um estudo realizado com acadêmicos de uma faculdade de medicina foi encontrado um resultado semelhante com os dados anteriormente citados, pois dos estudantes entrevistados do $1^{\circ}, 2^{\circ}, 3^{\circ}, 4^{\circ}$ anos e internato, 97\% destes praticavam a automedicação, sendo crescente a percentagem de uso dos medicamentos pelos alunos de acordo com a quantidade de anos estudados na faculdade, excetuando somente o período de internato. As percentagens são, respectivamente, $93,33 \%, 98,44 \%, 98,61 \%$ e $90 \%$. O mesmo estudo mostra que dos 280 alunos que argumentaram sobre a utilização de medicamentos que "sobraram" de prescrições anteriores, 135 deles afirmam praticar a automedicação por estes medicamentos (FONSECA et al., 2010).

Ao analisar o perfil dos

entrevistados em uma pesquisaficou evidenciado que o maior número de participantes era do sexo femininoe $66,5 \%$ alegaram a automedicação. O que pode ser explicado pelo contato muito maior que a mulher possui com medicamentos em todas as fases de sua vida em relação ao homem, uma maior procura por cuidados médicos e campanhas que são direcionadas a elas (AQUINO et al., 2010).

Em um estudo realizado por Dias et al. (2011) com respeito ao uso de psicotrópicos por parte dos profissionais de um determinado hospital fica evidente que lançam mão do uso dessa classe de fármacos para aliviar a tensão e estresse vivido no ambiente de trabalho. Fica percebido também que apesar do uso estão cientes de que essa é uma prática inadequada, principalmente, quando é referido o profissional de saúde como autor, pois ficou evidenciado que os profissionais entrevistados reconheciam as drogas psicoativas consumidascomo perigosas, mas que existia determinado autocontrole para o seu uso. Também relataram que tais drogas são de fácil acesso. $\mathrm{O}$ artigo evidencia também o equívoco dos profissionais quando justificam o uso de psicotrópicos para alívio dos sintomas supracitados, pois tais drogas produzem efeito letárgico que é divergente do efeito analgésico. 


\section{Tema 2: Estratégias para desconstrução da automedicação como prática segura}

Uma das várias maneiras de desconstruir a ideia (automedicação como uma prática segura) é ensinar durante a graduação aos acadêmicos que o consumo de medicamentos em si já possui certo grau de riscos, e que são ponderados durante o processo de prescrição de medicamentos. Assim, utilizá-los sem julgamento e auxílio profissional maximizam os ricos em número e potencial. Além disso, estes futuros profissionais da área da enfermagem devem ser instruídos que o conhecimento adquirido na disciplina de farmacologia não é suficiente para respaldar a prática de automedicação (SANTOS et al., 2012).

Para reverter este quadro Aquino et al. (2010) propõem da mesma forma supracitada ondese faz necessário a incorporação de práticaseducativas quanto ao uso correto dos medicamentos, riscos, benefícios, superdosagem, intoxicações, reações adversas, gastos para o sistema de saúde decorrentes de internações devido a problemas relacionados a medicamentos. Só assim, espera-se que a população possa receber cuidados de saúde de qualidade de profissionais competentes e preocupados em preservar a sua saúde.

$$
\begin{aligned}
& \text { Os analgésicos, ainda } \\
& \text { quegeralmente bem tolerados, são } \\
& \text { medicamentosapenas sintomáticos e não }
\end{aligned}
$$

isentos de risco, podendomascarar doenças subjacentes, exacerbarsintomas dolorosos, gerar enxaqueca crônica ecausar danos gástricos, hepáticos, e renais. Por isso, ressalta-se que o manejoda dor pode ser alcançado através de medidas não farmacológicas (LUZ et al., 2012).

Ainda como uma alternativa para combate, as instituições, onde trabalham profissionais que se automedicam, devem fazer busca ativa desses para identificação dos mesmos e propor meios que extingam a (s) causa (s) precipitante dessa atitude. Outrossim, deve ser feita avaliação periódica do estado de saúde emocional dos trabalhadores para que possa haver promoção da saúde e prevenção de doenças evitando assim, não só a automedicação, mas o absenteísmo e a falta de estímulo e bem-estar dor profissional em seu ambiente de trabalho. Todavia, existem profissionais que, apesar de enfrentarem problemas no seu cotidiano ocupacional, valorizam os princípios éticos da enfermagem e a promoção da saúde em sua concepção ampliadae utilizam artifícios para não se envolverem com os psicotrópicos como válvula de escape das pressões do trabalho e da vida como: ter bom relacionamento familiar;ter uma religião; ter um relacionamento amoroso ideal e seguro;ter oportunidade de trabalho; ter lazer; terboas amizades; procurar conhecimentos e informaçõesatravés de estudo; procurar ajuda 
de especialistas casonecessário; praticar esportes, entre outros (DIAS et al., 2011).

Este problema deve ser encarado de maneira séria e comprometedora por parte dos gestores de instituições onde estes profissionais trabalham e onde estão sendo formados profissionais da área da saúde para que seja formada uma consciência concreta sobre uso racional de medicamentos e seja reproduzida por estes através da educação em saúde (TOMASI et al., 2007).

\section{CONSIDERAÇÕES FINAIS}

O número de artigos encontrados provocou nos autores um desconforto por ser sua quantidade um número baixo e consideravelmente insuficiente pela relevância e atualidade do tema. Por isso, além desta investigação feita através de revisão literária, deve ser ampliado o estudo com relação à automedicação pelos sujeitos que deveriam combatê-la.

A automedicação está evidente e comprovada no meio dos profissionais e acadêmicos da área da saúde que deveriam ser o exemplo quanto ao manejo prudente dos fármacos. No entanto, deve ser considerada uma dimensão multifacetada que apresenta o problema, pois não é tão simples quanto se possa propor. A automedicação tornou-se uma via de escape para os profissionais e acadêmicos da área da saúde para o enfrentamento de problemas relacionados principalmente à sua profissão. Com isso, é previsível que haja em vários casos abusos ou erros quanto ao uso do medicamento, pois o fato de não procurarem ajuda devidamente habilitada possibilita maior chances de equívoco.

Evitar que isso aconteça num contexto complicado, como é o da saúde, torna-se necessárias medidas que estejam contemplando as delicadas questões biopsicossociais que envolvem a prática da automedicação, por isso o comprometimento não deve partir somente do profissional ou acadêmico, apesar destes terem sua parcela de responsabilidade, mas as instituições de ensino, de trabalho e governos devem trabalhar de forma a evitar este problema que não somente é praticada por profissionais, mas também pelo público em geral.

Por conseguinte, a ação preventiva da automedicação deve estar arraigada nas raízes da formação profissional dos atores da saúde, sendo incentivada por professores, instituições, mídia, entre outros.

\section{REFERÊNCIAS}

AQUINO, Daniela Silva de;BARROS, José Augusto Cabral de;SILVA, Maria Dolores Paes da.A automedicação e os acadêmicos da área de saúde.Ciência de saúde coletiva [periódico na Internet]. 2010 Ago [citado 2013 Ago 27]; 15 (5): 2533-2538. Disponível em: http://www.scielo.br/scielo.php?script=sci_art text\&pid=S1413$81232010000500027 \& \operatorname{lng}=$ pt. 
http://dx.doi.org/10.1590/S1413-

81232010000500027.

BAGGIO, Maria Aparecida; FORMAGGIO, Filomena Maria. Automedicação: desvelando o descuidado de si dos profissionais de enfermagem.Revista de enfermagem UERJ; 17(2): 224-228, abr.-jun. 2009. Article em Pt| LILACS | ID: 528344.

BRITO, Éverton Guedes de. Automedicação dos profissionais de saúde: uma revisão integrativa. Recife; s.n; 2010. 40 p. Thesis em Pt | LILACS | ID: 638496.

DIAS, Juliana Rodrigues Ferreira; ARAÚJO, Caroline Silva de; MARTINS, Elizabete Rose Costa; CLOS, Araci Carmen; FRANCISCO, Marcio Tadeu Ribeiro; SAMPAIO, Carlos Eduardo Peres. Fatores predisponentes ao uso próprio de psicotrópicos por profissionais de enfermagem.Revista de enfermermagem UERJ; 19(3): 445-451, jul.-set. 2011. Article em Pt | LILACS | ID: 618871.

FIGUEIREDO, Estevão Tavares de; MORAIS, Alline Moterani de; COSTA, Ana Maria Duarte Dias; TERRA, Fábio de Souza. Perfil dos estudantes de Medicina frente à automedicação para redução do peso.Revista da Sociedade Brasileira de Clínica Médica; 7(6)nov.-dez. 2009. Article em Pt | LILACS | ID: 533116.

FONSECA, Filipe Isper Rodrigues Meireles da; DEDIVITIS, Rogério Aparecido; SMOKOU, Andressa; LASCANE, Eduardo; CAVALHEIRO, Rubens Andrioli; RIBEIRO, Eduardo Fernandes; SILVA, Adilson Marques da; SANTOS, Edgard Bertolli dos. Frequência de automedicação entre estudantes da faculdade de medicina.Diagnóstico e tratamento; 15(2)abr.-jun. 2010. tab, ilus. Article em Pt |LILACS | ID: 550873.

LUZ, Tatiana Chama Borges;LUIZA, Vera Lucia;AVELAR, Fernando Genovez;HÖKERBERG, Yara Hahr Marques;PASSOS, Sonia Regina Lambert. Consumo de medicamentos por trabalhadores de hospital.Ciência de saúde coletiva [serial on the Internet]. 2012 Feb [cited 2013 Aug
27]; 17(2): 499-509. Available from:

http://www.scielo.br/scielo.php?script=sci_art text\&pid=S1413-

$81232012000200023 \& \operatorname{lng}=$ en .

http://dx.doi.org/10.1590/S1413-

81232012000200023.

SANTOS, Benedito dos; SOUZA, Letícia Gouveia de; DELGADO, Natália Matachun; TORRES, Wagner Oliveira. Incidência da automedicação em graduandos de enfermagem. Journal Health

ScienceInstitute; 30(2)abr.-jun. 2012. graf, tab. Article em Pt | LILACS | ID: 655210.

SOUZA, Layz Alves Ferreira;SILVA, Camila Damázio da;FERRAZ, Gisely

Carvalho;SOUSA, Fátima Aparecida Emm

Faleiros;PEREIRA, Lílian Varanda.

Prevalência e caracterização da prática de automedicação para alívio da dor entre estudantes universitários de enfermagem.Revista Latino-Americana Enfermagem [periódico na Internet]. 2011 Abr [citado 2013 Ago 27]; 19(2): 245-251. Disponível em:

http://www.scielo.br/scielo.php?script=sci_art text\&pid=S0104$11692011000200004 \& \operatorname{lng}=$ pt. http://dx.doi.org/10.1590/S010411692011000200004.

TOMASI, Elaine et al. Condições de trabalho e automedicação em profissionais da rede básica de saúde da zona urbana de Pelotas, RS. Revista brasileira deepidemiologia, São Paulo, v. 10, n. 1, Mar. 2007. Available from $<$ http://www.scielo.br/scielo.php?script=sci_a rttext\&pid=S1415-

$790 \times 2007000100008 \& \operatorname{lng}=$ en $\&$ nrm $=$ iso $>$. access on 29 Oct. 2013. http://dx.doi.org/10.1590/S1415790X2007000100008. 